\title{
DIEXTENSIONS ET PANEXTENSIONS DE CATÉGORIES
}

\author{
GEORGES HOFF
}

\begin{abstract}
Considering some different notions of kernel for a quotient functor, news kinds of extensions of (small) categories appear. In each context, one sees how 2-cocycles are naturally defined. Classical 2-cohomologies, like Hochschild's one (for which the need of commutativity is here discussed), are particular cases in this framework. One has thus an unifying point of view for extensions structures.
\end{abstract}

\section{Introduction}

Dans [9], nous reprenions la notion d'extension de (petites) catégories définie dans [8] pour l'élargir. De nombreux travaux ont montré le besoin réitéré et l'importance de ce type de structures donnant de la 2-cohomologie.

La définition considère le noyau $\mathbf{K}$ d'un foncteur quotient $\mathbf{H} \rightarrow \mathbf{C}$ i.e. plein (surjectif sur les morphismes) et bijectif sur les objets (par commodité, on peut dire que $\mathbf{H}$ et $\mathbf{C}$ ont les mêmes objets).

Définition 1.1. Une extension de catégories (de la catégorie $\mathbf{C}$ par la catégorie $\mathbf{K}$ ) est une suite $\mathbf{E}: \mathbf{K} \stackrel{i}{\longrightarrow} \mathbf{H} \stackrel{p}{\longrightarrow} \mathbf{C}$, où $i$ est un foncteur fidèle et $p$ un foncteur quotient, telle que pour $h$ et $h^{\prime} \in \mathbf{H}_{1}$, on a:

$$
p(h)=p\left(h^{\prime}\right) \Longleftrightarrow \exists ! k \in \mathbf{K}_{1}, h^{\prime}=i(k) h .
$$

Comme nous l'avons vu dans [9], ceci mène à de la 2-cohomologie à coefficients covariants et on peut l'adapter pour avoir de la 2-cohomologie à coefficients contravariants.

DÉFINITION 1.2. Une opextension de catégories est une suite $\mathbf{E}: \mathbf{K} \stackrel{i}{\longrightarrow}$ $\mathbf{H} \stackrel{p}{\longrightarrow} \mathbf{C}$, où $i$ est un foncteur fidèle et $p$ un foncteur quotient, telle que pour $h$ et $h^{\prime} \in \mathbf{H}_{1}$, on a:

$$
p(h)=p\left(h^{\prime}\right) \Longleftrightarrow \exists ! k \in \mathbf{K}_{1}, h^{\prime}=h i(k) .
$$

Received May 25, 2001. 
Hormis $\mathscr{G} r$ ou $\mathscr{A} b$, catégories des groupes ou des groupes abéliens, les catégories considérées sont petites. Si $\mathbf{C}$ est une catégorie, nous noterons $\mathbf{C}_{0}, \mathbf{C}_{1}, \mathbf{C}_{2}, \mathbf{C}_{3}, \ldots$ les ensembles de ses objets, de ses morphismes, de ses couples de morphismes composables... Si $A$ et $B \in \mathbf{C}_{0}$ sont des objets, nous noterons $\mathbf{C}(A, B)$ l'ensemble des morphismes $f$ de source $A$ et de but $B$ (on notera aussi $A=\alpha(f)$ et $B=\beta(f)$ ).

On remarquera, comme dans [9], que les extensions (resp. les opextensions) sont les précofibrations (resp. les préfibrations) au sens de Grothendieck dont les fibres sont des groupes. La catégorie $\mathbf{K}$ est donc un groupoïde coproduit (somme disjointe) des groupes fibres.

Dans les définitions ci-dessus, ce sont les fibres de $p$ qui déterminent la relation d'équivalence $p(h)=p\left(h^{\prime}\right)$ par une action à gauche ou à droite. Dans ce qui suit, nous remplacerons l'action sur $\mathbf{H}$ des groupes $\mathbf{K}_{A}=p^{-1}(A)$ indexés par $\mathbf{C}_{0}$ par celle de groupes $\mathbf{K}_{A, B}$ indexés par $\mathbf{C}_{0} \times \mathbf{C}_{0}$, puis par celle de groupes $\mathbf{K}_{f}$ indexés par $\mathbf{C}_{1}$. Ces manières différentes d'envisager le noyau d'un foncteur quotient fournissent de nouveaux types d'extensions.

La première démarche fait apparaitre de la 2-cohomologie à coefficients bivariants. Comme cas particulier, on retrouve la cohomologie de HochschildMitchell à coefficients dans un bimodule ([11]) et son interprétation en termes d'extensions. La seconde démarche prolonge naturellement la précédente et mène à une 2-cohomologie dont un cas particulier est la cohomologie de BauesWirsching à coefficients dans un système naturel ([2]). Nous mesurerons en passant le besoin de commutativité de ces contextes.

Ces nouvelles perspectives sur la 2-cohomologie s'étendent à l'étude des cohomologies d'ordres supérieurs à l'aide des extensions $n$-uples. Ceci fut envisagé par [3] et [6] avec nos extensions de [8] et, après la soumission du présent article, par [1] avec les extensions de [2].

L'auteur remercie M. C. Werquin pour son aimable $\mathrm{T}_{\mathrm{E}} \mathrm{X}$ assistance.

\section{Diextensions de categories}

Soit $\mathbf{H} \rightarrow \mathbf{C}$ un foncteur quotient entre (petites) catégories. Pour chaque couple d'objets $(A, B) \in \mathbf{C}_{0} \times \mathbf{C}_{0}\left(=\mathbf{H}_{0} \times \mathbf{H}_{0}\right)$, on considère un groupe $\mathbf{K}_{A, B}$. On définit ainsi un groupoïde

$$
\mathbf{K}=\coprod_{(A, B) \in \mathbf{C}_{0} \times \mathbf{C}_{0}} \mathbf{K}_{A, B}
$$

dont l'ensemble des objets $\mathbf{K}_{0}$ peut être identifié à $\mathbf{C}_{0} \times \mathbf{C}_{0}$.

DéfINITION 2.1. On dira que $\mathbf{K}$ opère fidèlement sur $\mathbf{H}$ si pour chaque $(A, B) \in \mathbf{C}_{0} \times \mathbf{C}_{0}$ on a:

$$
\mathbf{K}_{A, B} \times \mathbf{H}(A, B) \longrightarrow \mathbf{H}(A, B):(k, h) \mapsto k[h]
$$


de sorte que:

(i) $k^{\prime} k[h]=k^{\prime}[k[h]]$ et $1_{\mathbf{K}_{A, B}}[h]=h$,

(ii) si l'on a $k[h]=h^{\prime}$ et $k\left[h_{1}\right]=h_{1}^{\prime}$, alors on a

$$
k^{\prime}[h l]=h^{\prime} l \Longrightarrow k^{\prime}\left[h_{1} l\right]=h_{1}^{\prime} l \quad \text { et } \quad k^{\prime}[l h]=l h^{\prime} \Longrightarrow k^{\prime}\left[l h_{1}\right]=l h_{1}^{\prime} \text {, }
$$

quand ces expressions ont un sens.

REMARQUeS. Les expressions de (ii) ont un sens quand

$$
\beta(l)=A \quad \text { et } \quad k^{\prime} \in \mathbf{K}_{\alpha(l), B}, \quad \text { resp. } \quad \alpha(l)=B \quad \text { et } \quad k^{\prime} \in \mathbf{K}_{A, \beta(l)} \text {. }
$$

La condition de fidélité ( $i i$ ) dit que si un même $k \in \mathbf{K}_{1}$ égalise par son action deux couples $\left(h, h^{\prime}\right)$ et $\left(h_{1}, h_{1}^{\prime}\right)$, alors les couples de composés $\left(h l, h^{\prime} l\right)$ et $\left(h_{1} l, h_{1}^{\prime} l\right)$, resp. $\left(l h, l h^{\prime}\right)$ et $\left(l h_{1}, l h_{1}^{\prime}\right)$, sont égalisés par un même élément $k^{\prime}$ de $\mathbf{K}_{1}$. Elle étend naturellement une propriété qui est simple quand l'action est le fait de la composition par les éléments des fibres dans 1.1 et 1.2 (voir exemples ci-dessous).

Définition 2.2. Une diextension de catégories (de la catégorie $\mathbf{C}$ par la catégorie $\mathbf{K}$ ) est une suite $\mathbf{E}: \mathbf{K}--\rightarrow \mathbf{H} \stackrel{p}{\longrightarrow} \mathbf{C}$ où p est un foncteur quotient et où $\mathbf{K}$ opère fidèlement sur $\mathbf{H}$ de sorte que l'on ait:

$$
p(h)=p\left(h^{\prime}\right) \Longleftrightarrow \exists ! k \in \mathbf{K}_{1}, k[h]=h^{\prime} .
$$

EXEMPLES. Etant donnée une extension (resp. une opextension) de catégories au sens de 1.1 (resp. 1.2) $\mathbf{E}: \mathbf{K} \stackrel{i}{\longrightarrow} \mathbf{H} \stackrel{p}{\longrightarrow} \mathbf{C}$, en posant $\mathbf{K}_{A, B}=\mathbf{K}_{B}$ (resp. $\mathbf{K}_{A, B}=\mathbf{K}_{A}$ ) et $k[h]=i(k) h($ resp. $k[h]=h i(k))$, on obtient une diextension de catégories. Dans la première implication de 2.1 (ii), on a $k^{\prime}=k$ et dans la seconde $k^{\prime}$ est l'unique élément tel que $k^{\prime} l=l k$ (resp. $k^{\prime}=k$ dans la seconde implication et $k^{\prime}$ est l'unique élément tel que $l k^{\prime}=k l$ dans la première).

REmarques. On voit qu'une diextension n'est pas à la fois une extension et une opextension. Malgré le contexte bivariant qui va apparaître, on ne parle donc pas de biextensions. Signalons ici la notion d'appariement de fibrations considérée par [12] qui relie les constructions de [2] sur la cohomologie des catégories et celles de [5] qui décrit une cohomologie des diagrammes d'algèbres généralisant la cohomologie de Hochschild [7] dont nous reparlerons.

Proposition 2.3. Si l'on a $k[h]=k^{\prime}[h]$, alors on a $k=k^{\prime}$. Si l'on a $k[h]=h^{\prime}$, alors on a $h=k^{-1}\left[h^{\prime}\right]$. 
Preuve. Ces résultats sont conséquences, respectivement, de ( $\star$ ) de 2.2 et de (i) de 2.1.

Remarque. De par $(\star)$ de 2.2 , pour chaque $f \in \mathbf{C}_{1}$, le groupe $\mathbf{K}_{\alpha(f), \beta(f)}$ opère transitivement et effectivement, au sens de [2], sur l'ensemble $p^{-1}(f) \subset$ $\mathbf{H}_{1}$.

Proposition 2.4. Etant donnés $h \in \mathbf{H}_{1}$ et $k \in \mathbf{K}_{A, \alpha(h)}$ (resp. $k \in \mathbf{K}_{\beta(h), B}$ ), il existe un unique ${ }^{h} k \in \mathbf{K}_{1}$ (resp. $k^{h} \in \mathbf{K}_{1}$ ) tel que

$$
h k[l]={ }^{h} k[h l] \quad\left(\text { resp. } \quad k[l] h=k^{h}[l h]\right)
$$

lorsque ces expressions ont un sens (i.e. pour l et h composables).

Preuve. Du fait que l'on a $p(h l)=p(h k[l])$, resp. $p(l h)=p(k[l] h)$, l'existence et l'unicité de ${ }^{h} k$, resp. $k^{h}$, pour $l$ fixé, est conséquence de ( $\star$ ) de 2.2. De plus ceci ne dépend pas du choix de $l$ de par (ii) de 2.1. En effet, si l'on a $k[l]=l^{\prime}$ et $k\left[l_{1}\right]=l_{1}^{\prime}$, la seconde implication de (ii), où ${ }^{h} k$ joue le rôle de $k^{\prime}$ et $h$ celui de $l$, nous dit que l'on a:

$$
{ }^{h} k[h l]=h l^{\prime} \Rightarrow{ }^{h} k\left[h l_{1}\right]=h l_{1}^{\prime}
$$

et donc

$$
{ }^{h} k[h l]=h k[l] \Rightarrow{ }^{h} k\left[h l_{1}\right]=h k\left[l_{1}\right] .
$$

On a une même démonstration pour $k^{h}$.

THÉORÈME 2.5. L'opération $k \mapsto{ }^{h}\left(k^{h^{\prime}}\right)=\left({ }^{h} k\right)^{h^{\prime}}={ }^{h} k^{h^{\prime}}$ définit un foncteur $\mathbf{H} \times \mathbf{H}^{o p} \rightarrow \mathscr{G}$ r tel que $(A, B) \mapsto \mathbf{K}_{A, B}$.

Preuve. a) Comme on a, de par 2.4, les relations suivantes

$$
\begin{aligned}
& { }^{h}\left(k^{h^{\prime}}\right)\left[h l h^{\prime}\right]=h\left(k^{h^{\prime}}\right)\left[l h^{\prime}\right]=h k[l] h^{\prime}, \\
& \left({ }^{h} k\right)^{h^{\prime}}\left[h l h^{\prime}\right]={ }^{h} k[h l] h^{\prime}=h k[l] h^{\prime},
\end{aligned}
$$

l'unicité de 2.3 montre que l'on a ${ }^{h}\left(k^{h^{\prime}}\right)=\left({ }^{h} k\right)^{h^{\prime}}$.

b) Comme on a, de par 2.4, les relations suivantes:

$$
\begin{aligned}
& { }^{h}\left({ }^{h^{\prime}} k\right)\left[h h^{\prime} l\right]=h\left({ }^{h^{\prime}} k\right)\left[h^{\prime} l\right]=h\left(h^{\prime} k[l]\right)=\left(h h^{\prime}\right) k[l], \\
& \left(k^{h}\right)^{h^{\prime}}\left[l h h^{\prime}\right]=\left(k^{h}\right)[l h] h^{\prime}=(k[l] h) h^{\prime}=k[l]\left(h h^{\prime}\right),
\end{aligned}
$$

l'unicité de 2.4 montre que l'on a ${ }^{h}\left({ }^{\prime} k\right)={ }^{h h^{\prime}} k$ et $\left(k^{h}\right)^{h^{\prime}}=k^{h h^{\prime}}$ quand ces expressions ont un sens. 
c) Soit $1 \in \mathbf{H}_{1}$ une identité. On a les relations suivantes:

$$
\begin{aligned}
& { }^{1} k[1 h]=1 k[h]=k[h]=k[1 h], \\
& k^{1}[h 1]=k[h] 1=k[h]=k[h 1],
\end{aligned}
$$

et l'unicité de 2.3 montre alors que l'on a ${ }^{1} k=k^{1}=k$.

d) Il nous reste à démontrer que ${ }^{h}()^{h^{\prime}}: \mathbf{K}_{\beta\left(h^{\prime}\right), \alpha(h)} \rightarrow \mathbf{K}_{\alpha\left(h^{\prime}\right), \beta(h)}$ est un homomorphisme de groupes. Considérant les égalités:

$$
\begin{aligned}
\left({ }^{h} k\right)\left({ }^{h} k^{\prime}\right)[h l]={ }^{h} k\left[{ }^{h} k^{\prime}[h l]\right] & ={ }^{h} k\left[h k^{\prime}[l]\right] \\
& =h k\left[k^{\prime}[l]\right]=h\left(k k^{\prime}\right)[l]={ }^{h}\left(k k^{\prime}\right)[h l],
\end{aligned}
$$

de par l'unicité de 2.3 , on a l'égalité $\left({ }^{h} k\right)\left({ }^{h} k^{\prime}\right)={ }^{h}\left(k k^{\prime}\right)$. Ayant la même chose avec l'action à droite, avec a) ci -dessus, on a bien:

$$
{ }^{h}\left(k k^{\prime}\right){ }^{h^{\prime}}=\left({ }^{h} k^{h^{\prime}}\right)\left({ }^{h} k^{\prime h^{\prime}}\right) .
$$

THÉORÈME 2.6. On a $k^{\prime}\left[h^{\prime}\right] k[h]=k^{\prime \prime}\left[h^{\prime} h\right]$ avec:

$$
k^{\prime \prime}=\left(k^{\prime}\left[h^{\prime}\right] k\right)\left(k^{\prime h}\right)=\left(k^{\prime k[h]}\right)\left(h^{\prime} k\right),
$$

quand ces expressions ont un sens.

Preuve. On a les relations suivantes:

$$
\begin{aligned}
& k^{\prime}\left[h^{\prime}\right] k[h]=k^{\prime k[h]}\left[h^{\prime} k[h]\right]=k^{\prime k[h]}\left[{ }^{h^{\prime}} k\left[h^{\prime} h\right]\right]=\left(k^{\prime k[h]}\right)\left(h^{\prime} k\right)\left[h^{\prime} h\right], \\
& k^{\prime}\left[h^{\prime}\right] k[h]={ }^{k^{\prime}\left[h^{\prime}\right]} k\left[k^{\prime}\left[h^{\prime}\right] h\right]={ }^{k^{\prime}\left[h^{\prime}\right]} k\left[k^{\prime h}\left[h h^{\prime}\right]\right]=\left(k^{\prime}\left[h^{\prime}\right] k\right)\left(k^{\prime h}\right)\left[h^{\prime} h\right],
\end{aligned}
$$

et de par l'unicité de 2.3 issue de celle de ( $\star$ ) de 2.2, il vient la relation énoncée.

Remarques. Ce que l'on a dans 2.5 et 2.6 joue ici le même rôle que les bimultiplications permutables de [10] et que la propriété de distributivité de [2].

Proposition 2.7. Si on a une opération de $\mathbf{K} \operatorname{sur} \mathbf{H}$ :

$$
\mathbf{K}_{A, B} \times \mathbf{H}(A, B) \longrightarrow \mathbf{H}(A, B):(k, h) \mapsto k[h]
$$

telle que:

a) (i) de 2.1,

b) on a un foncteur $\mathbf{H} \times \mathbf{H}^{\text {op }} \stackrel{\Phi}{\longrightarrow} \mathscr{G}$ r tel que

$$
(A, B) \mapsto \mathbf{K}_{A, B} \quad \text { et } \quad\left(h, h^{\prime}\right) \mapsto{ }^{h}()^{h^{\prime}},
$$


c) 2.6 ,

d) $(\star) d e 2.2$.

Alors l'opération est fidèle.

Preuve. Supposons que l'on a $k[h]=h^{\prime}$ et $k\left[h_{1}\right]=h_{1}^{\prime}$, montrons que l'on a:

$$
k^{\prime}[h l]=h^{\prime} l \Longrightarrow k^{\prime}\left[h_{1} l\right]=h_{1}^{\prime},
$$

(la démonstration étant la même de l'autre côté). Ayant

$$
k^{\prime}[h l]=h^{\prime} l=k[h] 1[l],
$$

comme $\Phi$ est un foncteur, ${ }^{k[h]}$ ( ) est un homomorphisme de groupes et de par 2.6 on a:

$$
k^{\prime}=\left({ }^{k[h]} 1\right)\left(k^{l}\right)=1 k^{l}=k^{l} .
$$

Si on a aussi $k^{\prime \prime}\left[h_{1} l\right]=h_{1}^{\prime} l$, on a de même $k^{\prime \prime}=k^{l}$ et donc $k^{\prime \prime}=k^{\prime}$.

Remarque. Dans la définition 2.2 d'une diextension on peut donc remplacer (ii) par la donnée d'un foncteur $\mathbf{H} \times \mathbf{H}^{o p} \rightarrow \mathscr{G}_{r}$ vérifiant la propriété du théorème 2.6.

DÉfinition 2.8. Soit $M: \mathbf{C} \times \mathbf{C}^{o p} \rightarrow \mathscr{G}_{r}$ un foncteur, on appelle diextension de $C$ par $M$ une diextension de catégories $\mathbf{K} \rightarrow \mathbf{H} \stackrel{p}{\longrightarrow} \mathbf{C}$ telle que

$$
\mathbf{K}_{A, B}=M(A, B) \quad \text { et } \quad{ }^{h} k^{h^{\prime}}=M\left(p h, p h^{\prime}\right)(k) .
$$

\section{Diextensions et cocycles}

Considérons une diextension de catégories $\mathbf{E}: \mathbf{K}-\rightarrow \mathbf{H} \stackrel{p}{\longrightarrow} \mathbf{C}$.

DÉFINITIOn 3.1. Une section de la diextension $\mathbf{E}$ est la donnée, pour chaque $c \in \mathbf{C}_{1}$ d'un représentant $s(c)$ tel que $p s(c)=c$ de sorte que $s(c)$ soit une identité quand $c$ en est une.

Remarque. En associant à chaque $(A, B) \in \mathbf{C}_{0} \times \mathbf{C}_{0}$ le groupe $\mathbf{K}_{A, B}$ et à chaque $\left(c, c^{\prime}\right) \in \mathbf{C}_{1} \times \mathbf{C}_{1}$ le morphisme ${ }^{s(c)}()^{s\left(c^{\prime}\right)}$, on définit un coefficient de $\mathbf{C} \times \mathbf{C}^{o p}$ vers $\mathscr{G} r$ au sens de [9]. Ce n'est pas nécessairement un foncteur car $s$ n'en est pas nécessairement un.

THÉORÈme 3.2. La donnée d'une section s de $\mathbf{E}$ définit une application $\psi: \mathbf{C}_{2} \rightarrow \mathbf{K}_{1}$ telle que:

a) $\psi\left(c, c^{\prime}\right) \in \mathbf{K}_{\alpha\left(c^{\prime}\right), \beta(c)}$ et est une identité si c ou $c^{\prime}$ en est une, 
b) étant donné un triple composable $\left(c, c^{\prime}, c^{\prime \prime}\right) \in \mathbf{C}_{3}$, on a la relation:

$$
{ }^{s(c)} \psi\left(c^{\prime}, c^{\prime \prime}\right) \psi\left(c, c^{\prime} c^{\prime \prime}\right)=\left(\psi\left(c, c^{\prime}\right)^{s\left(c^{\prime \prime}\right)}\right) \psi\left(c c^{\prime}, c^{\prime \prime}\right),
$$

c) étant donnés $\left(c, c^{\prime}\right) \in \mathbf{C}_{2}, k \in \mathbf{K}_{1}$ et $h \in \mathbf{H}_{1}$, on a les relations:

$$
\begin{aligned}
& s(c) s\left(c^{\prime}\right) k=\left(\psi\left(c, c^{\prime}\right)^{k[h]}\right)\left({ }^{s\left(c c^{\prime}\right)} k\right)\left(\left(\psi\left(c, c^{\prime}\right)^{-1}\right)^{h}\right), \\
& k^{s(c) s\left(c^{\prime}\right)}=\left({ }^{k[h]} \psi\left(c, c^{\prime}\right)\right)\left(k^{s\left(c c^{\prime}\right)}\right)\left({ }^{h}\left(\psi\left(c, c^{\prime}\right)^{-1}\right)\right),
\end{aligned}
$$

quand ces expressions ont un sens.

Preuve. a) Comme on a $p s\left(c c^{\prime}\right)=p\left(s(c) s\left(c^{\prime}\right)\right)=c c^{\prime}$, la propriété $(\star)$ de 2.2 assure l'existence et l'unicité de $\psi\left(c, c^{\prime}\right)$ tel que $\psi\left(c, c^{\prime}\right)\left[s\left(c c^{\prime}\right)\right]=$ $s(c) s\left(c^{\prime}\right)$. Si $c$, resp. $c^{\prime}$, est une identité, on a $s\left(c c^{\prime}\right)=s\left(c^{\prime}\right)=s(c) s\left(c^{\prime}\right)$, resp. $s\left(c c^{\prime}\right)=s(c)=s(c) s\left(c^{\prime}\right)$ par définition de $s$. Dans ce cas, $\psi\left(c, c^{\prime}\right)$ est une identité de par (i) de 2.1 et l'unicité de 1.5 .

b) On a les égalités suivantes:

$$
\begin{aligned}
& s(c) s\left(c^{\prime}\right) s\left(c^{\prime \prime}\right)=s(c)\left(s\left(c^{\prime}\right) s\left(c^{\prime \prime}\right)\right)=s(c) \psi\left(c^{\prime}, c^{\prime \prime}\right)\left[s\left(c^{\prime} c^{\prime \prime}\right)\right] \\
&={ }^{s(c)} \psi\left(c^{\prime}, c^{\prime \prime}\right)\left[s(c) s\left(c^{\prime} c^{\prime \prime}\right)\right]={ }^{s(c)} \psi\left(c^{\prime}, c^{\prime \prime}\right)\left[\psi\left(c, c^{\prime} c^{\prime \prime}\right)\left[s\left(c\left(c^{\prime} c^{\prime \prime}\right)\right)\right]\right] \\
&={ }^{s(c)} \psi\left(c^{\prime}, c^{\prime \prime}\right) \psi\left(c, c^{\prime} c^{\prime \prime}\right)\left[s\left(c c^{\prime} c^{\prime \prime}\right)\right],
\end{aligned}
$$

$$
\begin{aligned}
s(c) s\left(c^{\prime}\right) s\left(c^{\prime \prime}\right)=\left(s(c) s\left(c^{\prime}\right)\right) s\left(c^{\prime \prime}\right) & =\psi\left(c, c^{\prime}\right)\left[s\left(c c^{\prime}\right)\right] s\left(c^{\prime \prime}\right) \\
\left.=\psi\left(c, c^{\prime}\right)^{s\left(c^{\prime \prime}\right)}\left[s\left(c c^{\prime}\right) s c^{\prime \prime}\right)\right]=\psi\left(c, c^{\prime}\right)^{s\left(c^{\prime \prime}\right)}\left[\psi\left(c c^{\prime}, c^{\prime \prime}\right) s\left(\left(c c^{\prime}\right) c^{\prime \prime}\right)\right] & \\
& =\left(\psi\left(c, c^{\prime}\right)^{s\left(c^{\prime \prime}\right)}\right) \psi\left(c c^{\prime}, c^{\prime \prime}\right)\left[s\left(c c^{\prime} c^{\prime \prime}\right)\right],
\end{aligned}
$$

et l'unicité de 2.3 permet de conclure.

c) On a $k \in \mathbf{K}_{A, B}, h \in \mathbf{H}(A, B)$ et dans la première expression $\alpha\left(c^{\prime}\right)=B$ alors que dans la seconde $\beta(c)=A$. Les formules de 2.4 et 2.6 permettent d'écrire les égalités suivantes:

$$
\begin{aligned}
s(c) s\left(c^{\prime}\right) k\left[s(c) s\left(c^{\prime}\right) h\right] & =s(c) s\left(c^{\prime}\right) k[h]=\psi\left(c, c^{\prime}\right)\left[s\left(c c^{\prime}\right)\right] k[h] \\
& =\left(\psi\left(c, c^{\prime}\right)^{k[h]}\right)\left(s\left(c c^{\prime}\right) k\right)\left[s\left(c c^{\prime}\right) h\right] \\
& =\left(\psi\left(c, c^{\prime}\right)^{k[h]}\right)\left({ }^{s\left(c c^{\prime}\right)} k\right)\left[\psi\left(c, c^{\prime}\right)^{-1}\left[s(c) s\left(c^{\prime}\right)\right] h\right] \\
& \left.=\left(\psi\left(c, c^{\prime}\right)^{k[h]}\right)\left({ }^{s\left(c c^{\prime}\right)} k\right) \psi\left(c, c^{\prime}\right)^{-1}\right)^{h}\left[s(c) s\left(c^{\prime}\right) h\right],
\end{aligned}
$$


et l'unicité de 2.3 donne le premier résultat. Le second provient de:

$$
\begin{aligned}
k^{s(c) s\left(c^{\prime}\right)}\left[h s(c) s\left(c^{\prime}\right)\right] & =k[h] s(c) s\left(c^{\prime}\right)=k[h] \psi\left(c, c^{\prime}\right)\left[s\left(c c^{\prime}\right)\right] \\
& =\left({ }^{k[h]} \psi\left(c, c^{\prime}\right)\right)\left(k^{s\left(c c^{\prime}\right)}\right)\left[h s\left(c c^{\prime}\right)\right] \\
& =\left({ }^{k[h]} \psi\left(c, c^{\prime}\right)\right)\left(k^{s\left(c c^{\prime}\right)}\right)\left[h \psi\left(c, c^{\prime}\right)^{-1}\left[s(c) s\left(c^{\prime}\right)\right]\right] \\
& =\left({ }^{k[h]} \psi\left(c, c^{\prime}\right)\right)\left(k^{s\left(c c^{\prime}\right)}\right)\left({ }^{h}\left(\psi\left(c, c^{\prime}\right)^{-1}\right)\right)\left[h s(c) s\left(c^{\prime}\right)\right] .
\end{aligned}
$$

DÉfINITION 3.3. L'application $\psi$ est appelée le 2-cocycle associé à la diextension $\mathbf{E}$ par la section $s$.

THÉORÈme 3.4. Soient $\psi$ et $\psi^{\prime}$ les 2-cocycles associés à $\mathbf{E}$ par des sections $s$ et $s^{\prime}$. Il existe une application $\tau: \mathbf{C}_{1} \rightarrow \mathbf{K}_{1}$ telle que

a) $\tau(c) \in \mathbf{K}_{\alpha(c), \beta(c)}$,

b) $\left.\psi\left(c, c^{\prime}\right)=\tau\left(c c^{\prime}\right)^{-1} \psi^{\prime}\left(c, c^{\prime}\right)\left(\tau(c)^{s^{\prime}\left(c^{\prime}\right)}\right){ }^{s(c)} \tau\left(c^{\prime}\right)\right)$

$$
=\tau\left(c c^{\prime}\right)^{-1} \psi^{\prime}\left(c, c^{\prime}\right)\left({ }^{s^{\prime}(c)} \tau\left(c^{\prime}\right)\right)\left(\tau(c)^{s\left(c^{\prime}\right)}\right) \quad \forall\left(c, c^{\prime}\right) \in \mathbf{C}_{2},
$$

c) ${ }^{s^{\prime}(c)} k=\left(\tau(c)^{k[h]}\right)\left({ }^{s(c)} k\right)\left(\tau(c)^{-1}\right)^{h}$ et $k^{s^{\prime}(c)}=\left({ }^{k[h]} \tau(c)\right)\left(k^{s(c)}\right)\left({ }^{h}\left(\tau(c)^{-1}\right)\right)$,

quand ces expressions ont un sens.

Preuve. a) Comme $p s(c)=p s^{\prime}(c)=c$, la propriété ( $\star$ ) de 2.2 assure l'existence et l'unicité de $\tau(c) \in \mathbf{K}_{\alpha(c), \beta(c)}$ tel que $s^{\prime}(c)=\tau(c)[s(c)]$.

b) On utilise encore la formule de 2.6 et l'unicité de 2.3 avec

$$
s^{\prime}\left(c c^{\prime}\right)=\tau\left(c c^{\prime}\right)\left[s\left(c c^{\prime}\right)\right]
$$

d'une part, et

$$
\begin{aligned}
s^{\prime}\left(c c^{\prime}\right) & =\psi^{\prime}\left(c, c^{\prime}\right)\left[s^{\prime}(c) s^{\prime}\left(c^{\prime}\right)\right]=\psi^{\prime}\left(c, c^{\prime}\right)\left[\tau(c)[s(c)] \tau\left(c^{\prime}\right)\left[s\left(c^{\prime}\right)\right]\right] \\
& =\psi^{\prime}\left(c, c^{\prime}\right)\left[\left(\tau(c)^{s^{\prime}\left(c^{\prime}\right)}\right)\left({ }^{s(c)} \tau\left(c^{\prime}\right)\right)\left[s(c) s\left(c^{\prime}\right)\right]\right] \\
& =\psi^{\prime}\left(c, c^{\prime}\right)\left(\tau(c)^{s^{\prime}\left(c^{\prime}\right)}\right)\left({ }^{s(c)} \tau\left(c^{\prime}\right)\right) \psi\left(c, c^{\prime}\right)^{-1}\left[s\left(c c^{\prime}\right)\right],
\end{aligned}
$$

d'autre part pour la première égalité. La formule de 2.6 donne aussi $\left.{ }^{s^{\prime}(c)} \tau\left(c^{\prime}\right)\right)\left(\tau(c)^{s\left(c^{\prime}\right)}\right)$ au milieu du calcul précédent et donc la deuxième égalité.

c) L'unicité de 2.3 donne les derniers résultats en considérant:

$$
\begin{aligned}
s^{\prime}(c) k\left[s^{\prime}(c) h\right] & =s^{\prime}(c) k[h]=\tau(c)[s(c)] k[h]=\left(\tau(c)^{k[h]}\right)\left({ }^{s(c)} k\right)[s(c) h] \\
& =\left(\tau(c)^{k[h]}\right)\left({ }^{s(c)} k\right)\left[\tau(c)^{-1}\left[s^{\prime}(c)\right] h\right] \\
& =\left(\tau(c)^{k[h]}\right)\left({ }^{s(c)} k\right)\left(\tau(c)^{-1}\right)^{h}\left[s^{\prime}(c) h\right],
\end{aligned}
$$




$$
\begin{aligned}
k^{s^{\prime}(c)}\left[h s^{\prime}(c)\right] & =k[h] s^{\prime}(c)=k[h] \tau(c)[s(c)]=\left({ }^{k[h]} \tau(c)\right)\left(k^{s(c)}\right)[h s(c)] \\
& =\left({ }^{k[h]} \tau(c)\right)\left(k^{s(c)}\right)\left[h \tau(c)^{-1}\left[s^{\prime}(c)\right]\right] \\
& =\left({ }^{k[h]} \tau(c)\right)\left(k^{s(c)}\right)\left({ }^{h}\left(\tau(c)^{-1}\right)\right)\left[h s^{\prime}(c)\right] .
\end{aligned}
$$

Remarque. Les conditions c) de 3.2 et 3.4, comme 2.6 se ramènent à des conditions de commutativité quand $\mathbf{E}$ est une diextension de $\mathbf{C}$ par un foncteur $M: \mathbf{C} \times \mathbf{C}^{o p} \rightarrow \mathscr{G} r$ et disparaît si $M$ est à valeurs dans $\mathscr{A} b$, i.e. si $M$ est un C-bimodule.

Définition 3.5. Deux diextensions de catégories $\mathbf{E}: \mathbf{K} \rightarrow \mathbf{H} \stackrel{p}{\longrightarrow} \mathbf{C}$ et $\mathbf{E}^{\prime}: \mathbf{K}^{\prime} \rightarrow \mathbf{H}^{\prime} \stackrel{p^{\prime}}{\longrightarrow} \mathbf{C}$ sont dites congrues s'il existe un foncteur inversible $\mu: \mathbf{H} \rightarrow \mathbf{H}^{\prime}$ tel que

a) $p^{\prime} \mu=p$,

b) $k[\mu(h)]=\mu(k[h])$ quand ceci a un sens.

On notera Diext $(\mathbf{C}, \mathbf{K})$, resp. Diext $(\mathbf{C}, M)$, l'ensemble des classes de congruence de diextensions de $\mathbf{C}$ par un groupoïde $\mathbf{K}$, resp. un foncteur $M: \mathbf{C} \times \mathbf{C}^{o p} \rightarrow$ $\mathscr{G} r$.

Lemme 3.6. Dans les conditions de la définition 3.5, on a:

$$
{ }^{h} k^{h^{\prime}}={ }^{\mu(h)} k^{\mu\left(h^{\prime}\right)}
$$

quand ceci a un sens.

Preuve. Considérons $(h, l) \in \mathbf{H}_{2}$, on a

$$
\begin{aligned}
{ }^{\mu(h)} k[\mu(h l)] & ={ }^{\mu(h)} k[\mu(h) \mu(l)]=\mu(h) k[\mu(l)]=\mu(h) \mu(k[l]) \\
& =\mu(h k[l])=\mu\left({ }^{h} k[h l]\right)={ }^{h} k[\mu(h l)] .
\end{aligned}
$$

De par l'unicité de 2.3 , on a donc ${ }^{\mu(h)} k={ }^{h} k$ et de même $k^{\mu(h)}=k^{h}$ quand ces expressions ont un sens.

Remarque. Cette propriété est triviale dans le cas où $\mathbf{E}$ est une diextension par un foncteur $M: \mathbf{C} \times \mathbf{C}^{o p} \rightarrow \mathscr{G} r$.

ThÉorème 3.7. Soient $\mathbf{E}$ et $\mathbf{E}^{\prime}$ deux diextensions de $\mathbf{C}$ par $\mathbf{K}$. Si $\mathbf{E}$ et $\mathbf{E}^{\prime}$ sont congrues et si $\psi$ est un 2-cocycle associé à $\mathbf{E}$, alors $\psi$ est aussi un 2-cocycle associé à $\mathbf{E}^{\prime}$. Réciproquement, si un même 2-cocycle $\psi$ est associé à $\mathbf{E}$ et à $\mathbf{E}^{\prime}$, alors $\mathbf{E}$ et $\mathbf{E}^{\prime}$ sont congrues. 
Preuve. a) Soit $s$ la section qui associe $\psi$ à E. En posant $s^{\prime}(c)=\mu(s(c))$, on définit une section $s^{\prime}$ de $\mathbf{E}^{\prime}$. Comme $\psi$ est associé à $s$, on a

$$
\psi\left(c, c^{\prime}\right)\left[s\left(c c^{\prime}\right)\right]=s(c) s\left(c^{\prime}\right) \quad \text { et } \quad \mu\left(\psi\left(c, c^{\prime}\right)\left[s\left(c c^{\prime}\right)\right]\right)=\mu\left(s(c) s\left(c^{\prime}\right)\right) .
$$

De par 3.5, on a alors

$$
\mu\left(\psi\left(c, c^{\prime}\right)\left[s\left(c c^{\prime}\right)\right]\right)=\psi\left(c, c^{\prime}\right)\left[\mu\left(s\left(c c^{\prime}\right)\right)\right]=\psi\left(c, c^{\prime}\right)\left[s^{\prime}\left(c c^{\prime}\right)\right],
$$

et

$$
\mu\left(s(c) s\left(c^{\prime}\right)\right)=\mu\left(s(c) \mu\left(s\left(c^{\prime}\right)\right)=s^{\prime}(c) s^{\prime}\left(c^{\prime}\right) .\right.
$$

Ceci montre que $\psi$ est associé à la section $s^{\prime}$.

b) Soient $s$ et $s^{\prime}$ les sections qui associent $\psi$ à $\mathbf{E}$ et $\mathbf{E}^{\prime}$ respectivement. Un morphisme $h \in \mathbf{H}_{1}$ s'écrit de manière unique sous la forme $k[s(p h)]$ avec $k \in \mathbf{K}_{1}$. En posant $\mu(h)=k\left[s^{\prime}(p h)\right]$, on va définir un foncteur $\mu: \mathbf{H} \rightarrow \mathbf{H}^{\prime}$ qui fait de $\mathbf{E}$ et $\mathbf{E}^{\prime}$ des extensions congrues. Comme on a

$$
p^{\prime}\left(\mu((h))=p^{\prime}\left(k\left[s^{\prime}(p h)\right]=p^{\prime}\left(s^{\prime}(p h)\right)=p h,\right.\right.
$$

on a a) de 3.5. Pour b) de 3.5, en posant $h=k[s(p h)]$, on a

$$
\begin{aligned}
k^{\prime}[\mu(h)] & =k^{\prime}[\mu(k[s(p h)])]=k^{\prime}\left[k\left[s^{\prime}(p h)\right]\right]=k^{\prime} k\left[s^{\prime}(p h)\right] \\
& =\mu\left(k^{\prime} k[s(p h)]\right)=\mu\left(k^{\prime}[k[s(p h)]]\right)=\mu\left(k^{\prime}[h]\right) .
\end{aligned}
$$

Il reste à montrer que $\mu$ est un foncteur. On a évidemment $\mu(1)=1$ et on va montrer que l'on a $\mu\left(h h^{\prime}\right)=\mu(h) \mu\left(h^{\prime}\right)$. Soient $h=k[s(p h)]$ et $h^{\prime}=$ $k^{\prime}\left[s\left(p h^{\prime}\right)\right]$ composables, on a $\mu(h)=k\left[s^{\prime}(p h)\right]$ et $\mu\left(h^{\prime}\right)=k^{\prime}\left[s^{\prime}\left(p h^{\prime}\right)\right]$. Il vient alors

$$
\begin{aligned}
\mu(h) \mu\left(h^{\prime}\right) & =k\left[s^{\prime}(p h)\right] k^{\prime}\left[s^{\prime}\left(p h^{\prime}\right)\right] \\
& =\left(^{k\left[s^{\prime}(p h)\right]} k^{\prime}\right)\left(k^{s^{\prime}\left(p h^{\prime}\right)}\right)\left[s^{\prime}(p h) s^{\prime}\left(p h^{\prime}\right)\right] \\
& =\left(^{k\left[s^{\prime}(p h)\right]} k^{\prime}\right)\left(k^{s^{\prime}\left(p h^{\prime}\right)}\right)\left[\psi\left(p h, p h^{\prime}\right)\left[s^{\prime}\left(p h p h^{\prime}\right)\right]\right] \\
& =\left({ }^{\mu(k[s(p h)]} k^{\prime}\right)\left(k^{\mu\left(s\left(p h^{\prime}\right)\right)}\right) \psi\left(p h, p h^{\prime}\right)\left[\mu\left(s\left(p h p h^{\prime}\right)\right)\right] \\
& =\mu\left(\left({ }^{k[s(p h)]} k^{\prime}\right)\left(k^{s\left(p h^{\prime}\right)}\right) \psi\left(p h, p h^{\prime}\right)\left[s\left(p h p h^{\prime}\right)\right]\right) \\
& =\mu\left(\left({ }^{k[s(p h)]} k^{\prime}\right)\left(k^{s\left(p h^{\prime}\right)}\right)\left[s(p h) s\left(p h^{\prime}\right)\right]\right) \\
& =\mu\left(k[s(p h)] k^{\prime}\left[s\left(p h^{\prime}\right)\right]\right)=\mu\left(h h^{\prime}\right),
\end{aligned}
$$

en utilisant successivement 2.6, (i) de 2.1, 3.5 démontré précédemment et 3.6 ainsi que le fait que $\psi$ est associé par $s$ et par $s^{\prime}$ aux deux extensions considérées. L'inverse de $\mu$ est défini de la même manière. 


\section{Cohomologie à coefficients bivariants}

Considérons une (petite) catégorie $\mathbf{C}$, un foncteur $M: \mathbf{C} \times \mathbf{C}^{o p} \rightarrow \mathscr{G} r$ et le groupoîde

$$
\mathbf{K}=\coprod_{(A, B) \in \mathbf{C}_{0} \times \mathbf{C}_{0}} M(A, B) .
$$

On notera $\mathbf{K}_{A, B}=M(A, B)$ et ${ }^{c} k^{c^{\prime}}=M\left(c, c^{\prime}\right)(k)$.

Proposition 4.1. Si l'on a une diextension de $\mathbf{C}$ par $M$, alors $M$ est un C-bimodule.

Preuve. Ayant une diextension, de par 2.6 on a

$$
\left(k^{\prime}\left[h^{\prime}\right] k\right)\left(k^{\prime h}\right)=\left(k^{\prime k[h]}\right)\left(h^{\prime} k\right)
$$

quand ces expressions ont un sens. Comme la diextension considérée est une diextension de $\mathbf{C}$ par $M$, de par 2.8 on a de plus

$$
k^{\prime}\left[h^{\prime}\right] k={ }^{p h^{\prime}} k, \quad k^{\prime h}=k^{p h}, \quad k^{\prime k[h]}=k^{\prime p h}, \quad h^{\prime} k={ }^{p h^{\prime}} k .
$$

Soient $k$ et $k^{\prime} \in \mathbf{K}_{A, B}$, on a donc

$$
\left({ }^{1_{A}} k\right)\left(k^{\prime 1_{B}}\right)=\left(k^{\prime 1_{B}}\right)\left({ }^{1_{A}} k\right) \quad \text { i.e. } \quad k k^{\prime}=k^{\prime} k,
$$

ce qu'il fallait démontrer.

Dans la suite on considère un $\mathbf{C}$-bimodule $M$.

Définition 4.2. Un 2-cocycle de $C$ vers $M$ est la donnée d'une application $\psi: \mathbf{C}_{1} \rightarrow \mathbf{K}_{1}$ telle que:

a) $\psi\left(c, c^{\prime}\right) \in \mathbf{K}_{\alpha\left(c^{\prime}\right), \beta(c)}$ et est une identité si c ou c' en est une,

b) ${ }^{c} \psi\left(c^{\prime}, c^{\prime \prime}\right) \psi\left(c, c^{\prime} c^{\prime \prime}\right)=\left(\psi\left(c, c^{\prime}\right)^{c^{\prime \prime}}\right) \psi\left(c c^{\prime}, c^{\prime \prime}\right) \quad \forall\left(c, c^{\prime}, c^{\prime \prime}\right) \in \mathbf{C}_{3}$.

On notera les différences avec 3.2 comme expliqué dans la remarque suivant le théorème 3.4 et selon la proposition 4.1.

Définition 4.3. Deux cocycles $\psi$ et $\psi^{\prime}$ de $\mathbf{C}$ vers $M$ sont dits cohomologues s'il existe une application $\tau: \mathbf{C}_{1} \rightarrow \mathbf{K}_{1}$ telle que:

a) $\tau(c) \in \mathbf{K}_{\alpha(c), \beta(c)}$,

b) $\psi\left(c, c^{\prime}\right)=\tau\left(c c^{\prime}\right)^{-1} \psi^{\prime}\left(c, c^{\prime}\right)\left(\tau(c)^{c^{\prime}}\right)\left({ }^{c} \tau\left(c^{\prime}\right)\right)$

$$
=\tau\left(c c^{\prime}\right)^{-1} \psi^{\prime}\left(c, c^{\prime}\right)\left({ }^{c} \tau\left(c^{\prime}\right)\right)\left(\tau(c)^{c^{\prime}}\right), \quad \forall\left(c, c^{\prime}\right) \in \mathbf{C}_{2} .
$$

Proposition 4.4. La cohomologie ainsi définie est une relation d'équivalence sur l'ensemble des 2-cocycles de $\mathbf{C}$ vers $M$. 
Preuve. Un cocycle est cohomologue à lui-même avec $\tau(c)=1_{\mathbf{K}_{\alpha(c), \beta(c)}}$. Si $\psi$ et $\psi^{\prime}$ sont cohomologues de par $\tau$, en posant $\tau^{\prime}(c)=\tau(c)^{-1}$ on a $\psi^{\prime}$ et $\psi$ cohomologues. Supposons que $\psi$ et $\psi^{\prime}$ soient cohomologues de par $\tau$ et que $\psi^{\prime}$ et $\psi^{\prime \prime}$ le soient de par $\tau^{\prime}$. On pose $\tau^{\prime \prime}(c)=\tau^{\prime}(c) \tau(c)$ et il vient:

$$
\psi\left(c, c^{\prime}\right)=\tau\left(c c^{\prime}\right)^{-1} \tau^{\prime}\left(c c^{\prime}\right)^{-1} \psi^{\prime \prime}\left(c, c^{\prime}\right)\left(\tau^{\prime}(c)^{c^{\prime}}\right)\left({ }^{c} \tau^{\prime}\left(c^{\prime}\right)\right)\left(\tau(c)^{c^{\prime}}\right)\left({ }^{c} \tau\left(c^{\prime}\right)\right) .
$$

Utilisant la commutativité impliquée par 4.1, il vient:

$$
=\tau\left(c c^{\prime}\right)^{-1} \tau^{\prime}\left(c c^{\prime}\right)^{-1} \psi^{\prime \prime}\left(c, c^{\prime}\right)\left(\tau^{\prime}(c)^{c^{\prime}}\right)\left(\tau(c)^{c^{\prime}}\right)\left({ }^{c} \tau^{\prime}\left(c^{\prime}\right)\right)\left({ }^{c} \tau\left(c^{\prime}\right)\right) .
$$

Et comme ${ }^{c}()$ et ()$^{c^{\prime}}$ sont des homomorphismes de groupes:

$$
\psi\left(c, c^{\prime}\right)=\tau^{\prime \prime}\left(c c^{\prime}\right)^{-1} \psi\left(c, c^{\prime}\right)\left(\tau^{\prime \prime}(c)^{c^{\prime}}\right)\left({ }^{c} \tau^{\prime \prime}\left(c^{\prime}\right)\right),
$$

explicite la cohomologie de $\psi$ et $\psi^{\prime \prime}$.

Définition 4.5. L'ensemble des classes de cohomologie de 2-cocycles de $\mathbf{C}$ vers $M$ est appelé l'ensemble de cohomologie de dimension 2 de $\mathbf{C}$ vers $M$ et est noté $H^{2}(\mathbf{C}, M)$.

THÉORÈME 4.6. L'application, qui à chaque diextension de $\mathbf{C}$ par M associe la classe de cohomologie d'un de ses 2-cocycles associés, définit une bijection

$$
\omega: \operatorname{Diext}(\mathbf{C}, M) \longrightarrow H^{2}(\mathbf{C}, M) .
$$

Preuve. a) Le théorème 3.2 montre comment à une diextension $\mathbf{E}$ on associe un 2-cocycle et le théorème 3.4 montre que la classe de cohomologie de celui-ci ne dépend que de $\mathbf{E}$. Le théorème 3.7 montre qu'à deux diextensions congrues on associe le même élément de $H^{2}(\mathbf{C}, M)$. L'application $\omega$ est donc bien définie.

b) Soient $\mathbf{E}$ et $\mathbf{E}^{\prime}$ deux extensions auxquelles sont associés des 2-cocycles $\psi$ et $\psi^{\prime}$ cohomologues définis par des sections $s$ et $s^{\prime}$ respectivement. On définit une nouvelle section $s_{1}$ de $\mathbf{E}$ par $s_{1}(c)=\tau(c)^{-1}[s(c)]$. Le cocycle $\psi_{1}$ défini par $s_{1}$ vérifie alors

$$
\psi_{1}\left(c, c^{\prime}\right)=\tau\left(c c^{\prime}\right)^{-1} \psi\left(c, c^{\prime}\right)\left(\tau(c)^{c^{\prime}}\right)\left({ }^{c} \tau\left(c^{\prime}\right)\right)
$$

et l'application $k\left[s_{1}(c)\right] \mapsto k\left[s^{\prime}(c)\right]$ définit une congruence de $\mathbf{E}$ vers $\mathbf{E}^{\prime}$. Ceci montre que $\omega$ est injective.

c) Soit $\psi$ un 2-cocycle de $\mathbf{C}$ vers $M$. nous allons construire une diextension à laquelle $\psi$ est associé. Ceci montrera que $\omega$ est surjective et finira la preuve. 
Soit $\mathbf{H}_{\psi}$ la catégorie dont les morphismes sont les paires $(c, k)$ avec $c \in \mathbf{C}_{1}$ et $k \in \mathbf{K}_{\alpha(c), \beta(c)}$ telles que

$\alpha(c, k)=\alpha(c), \quad \beta(c, k)=\beta(c), \quad(c, k)\left(c^{\prime}, k^{\prime}\right)=\left(c c^{\prime},\left({ }^{c} k^{\prime}\right)\left(k^{c^{\prime}}\right) \psi\left(c, c^{\prime}\right)\right)$.

Vérifions que cette composition est associative.

$$
\begin{aligned}
\left((c, k)\left(c^{\prime}, k^{\prime}\right)\right)\left(c^{\prime \prime}, k^{\prime \prime}\right) & =\left(c c^{\prime},\left({ }^{c} k^{\prime}\right)\left(k^{c^{\prime}}\right) \psi\left(c, c^{\prime}\right)\right)\left(c^{\prime \prime}, k^{\prime \prime}\right) \\
& =\left(c c^{\prime} c^{\prime \prime},\left({ }^{c c^{\prime}} k^{\prime \prime}\right)\left(\left({ }^{c} k^{\prime}\right) k^{c^{\prime}} \psi\left(c, c^{\prime}\right)\right)^{c^{\prime \prime}} \psi\left(c c^{\prime}, c^{\prime \prime}\right)\right) \\
& =\left(c c^{\prime} c^{\prime \prime},\left({ }^{c c^{\prime}} k^{\prime \prime}\right)\left({ }^{c} k^{\prime c^{\prime \prime}}\right)\left(k^{c^{\prime} c^{\prime \prime}}\right)\left(\psi\left(c, c^{\prime}\right) c^{\prime \prime}\right) \psi\left(c c^{\prime}, c^{\prime \prime}\right)\right) \\
& =\left(c c^{\prime} c^{\prime \prime},\left({ }^{c c^{\prime}} k^{\prime \prime}\right)\left({ }^{c} k^{\prime c^{\prime \prime}}\right)\left(k^{c^{\prime} c^{\prime \prime}}\right)\left({ }^{c} \psi\left(c^{\prime}, c^{\prime \prime}\right)\right) \psi\left(c, c^{\prime} c^{\prime \prime}\right)\right) \\
& =\left(c c^{\prime} c^{\prime \prime},\left({ }^{c c^{\prime}} k^{\prime \prime}\right)\left({ }^{c} k^{\prime c^{\prime \prime}}\right)\left({ }^{c} \psi\left(c^{\prime}, c^{\prime \prime}\right)\right)\left(k^{c^{\prime} c^{\prime \prime}}\right) \psi\left(c, c^{\prime} c^{\prime \prime}\right)\right) \\
& =\left(c c^{\prime} c^{\prime \prime},{ }^{c}\left(\left(^{\prime} k^{\prime \prime}\right)\left(k^{\prime c^{\prime \prime}}\right) \psi\left(c^{\prime}, c^{\prime \prime}\right)\right)\left(k^{c^{\prime} c^{\prime \prime}}\right) \psi\left(c, c^{\prime} c^{\prime \prime}\right)\right) \\
& \left.=(c, k)\left(c^{\prime} c^{\prime \prime}, c^{c^{\prime}} k^{\prime \prime}\right)\left(k^{\prime c^{\prime \prime}}\right) \psi\left(c^{\prime}, c^{\prime \prime}\right)\right) \\
& =(c, k)\left(\left(c^{\prime}, k^{\prime}\right)\left(c^{\prime \prime}, k^{\prime \prime}\right)\right) .
\end{aligned}
$$

Avec $p(c, k)=c$ et $k\left[\left(c, k^{\prime}\right)\right]=\left(c, k k^{\prime}\right)$ on a une diextension de $\mathbf{C}$ par $M$ et $\psi$ est le 2-cocycle associé à la section définie par $s(c)=\left(c, 1_{\mathbf{K}_{\alpha(c), \beta(c)}}\right)$.

La proposition 4.1 permet de voir le besoin en commutativité de la cohomologie à coefficients bivariants. Le théorème précédent fournit une interprétation en termes d'extensions de la 2-cohomologie de Hochschild-Mitchell évoquée par Mitchell [11] et Baues-Wirsching [2].

Hochschild [7] a introduit ce type de cohomologie (bivariante) dans un cadre commutatif pour l'étude des algèbres associatives. Ceci a été généralisé par Gerstenhaber et Schack [5] qui ont décrit une cohomologie des diagrammes d'algèbres.

Hormis ce que nous en avons dit en 2 et 3 , le cadre non abélien a été abordé par Dedecker et Lue pour les algèbres dans [4] et [10].

\section{Panextensions de catégories}

Les ingrédients techniques de ce qui suit étant contenus dans ce que nous avons $\mathrm{vu}$, nous omettrons les démonstrations qui sont des adaptations de celles déjà faites.

Soient $\mathbf{C}$ une (petite) catégorie et $p: \mathbf{H} \rightarrow \mathbf{C}$ un foncteur quotient. Pour chaque morphisme $f \in \mathbf{C}_{1}$, on considère un groupe $\mathbf{K}_{f}$. On définit ainsi un groupoïde

$$
\mathbf{K}=\coprod_{f \in \mathbf{C}_{1}} \mathbf{K}_{f}
$$


dont l'ensemble des objets peut être identifié à $\mathbf{C}_{1}$.

DéfInItION 5.1. On dira que $\mathbf{K}$ opère fidèlement sur $\mathbf{H}$ si pour chaque $f \in \mathbf{C}_{1}$ on a:

$$
\mathbf{K}_{f} \times p^{-1}(f) \longrightarrow p^{-1}(f):(k, h) \mapsto k[h]
$$

de sorte que:

(i) $k^{\prime} k[h]=k^{\prime}[k[h]]$ et $1_{\mathbf{K}_{f}}[h]=h$,

(ii) si l'on a $k[h]=h^{\prime}$ et $k\left[h_{1}\right]=h_{1}^{\prime}$, alors on a

$$
k^{\prime}[h l]=h^{\prime} l \Longrightarrow k^{\prime}\left[h_{1} l\right]=h_{1}^{\prime} l \quad \text { et } \quad k^{\prime}[l h]=l h^{\prime} \Longrightarrow k^{\prime}\left[l h_{1}\right]=l h_{1}^{\prime},
$$

quand ces expressions ont un sens.

Définition 5.2. Une panextension de catégories (de la catégorie $\mathbf{C}$ par la catégorie $\mathbf{K}$ ) est une suite $\mathbf{E}: \mathbf{K} \rightarrow \mathbf{H} \stackrel{p}{\longrightarrow} \mathbf{C}$, où $p$ est un foncteur quotient (i.e. plein et bijectif sur les objets) et où $\mathbf{K}$ opère fidèlement sur $\mathbf{H}$ de sorte que l'on ait:

$$
p(h)=p\left(h^{\prime}\right) \Longleftrightarrow \exists ! k \in \mathbf{K}_{1}, k[h]=h^{\prime} .
$$

Exemples. Etant donnée une diextension de catégories $\mathbf{E}: \mathbf{K} \rightarrow \mathbf{H} \stackrel{p}{\longrightarrow} \mathbf{C}$, en posant $\mathbf{K}_{f}=\mathbf{K}_{\alpha(f), \beta(f)}$ on obtient une panextension de catégories. On verra aussi que les extensions linéaires de Baues-Wirsching ([2]) sont des panextensions.

Proposition 5.3. Si l'on a $k[h]=k^{\prime}[h]$, alors on a $k=k^{\prime}$. Si l'on a $k[h]=h^{\prime}$, alors on a $h=k^{-1}\left[h^{\prime}\right]$.

Proposition 5.4. Etant donnés $h \in \mathbf{H}_{1}$ et $k \in \mathbf{K}_{f}$ avec $\alpha(h)=\beta(f)$ (resp. $\beta(h)=\alpha(f)$ ), il existe un unique ${ }^{h} k \in \mathbf{K}_{1}$ (resp. $k^{h} \in \mathbf{K}_{1}$ ) tel que

$$
h k[l]={ }^{h} k[h l] \quad\left(\text { resp. } \quad k[l] h=k^{h}[l h]\right)
$$

lorsque ces expressions ont un sens.

RAPPEL. Comme [2], nous noterons $F \mathbf{C}$ la catégorie des factorisations de $\mathbf{C}$ dont les objets sont les morphismes de $\mathbf{C}$ et où les morphismes $f \rightarrow g$ sont les couples $(u, v)$ de morphismes de $\mathbf{C}$ tels que $g=u f v$, la composition étant définie par $(u, v)\left(u^{\prime}, v^{\prime}\right)=\left(u u^{\prime}, v^{\prime} v\right)$. On a le foncteur d'oubli $F \mathbf{C} \rightarrow \mathbf{C} \times \mathbf{C}^{o p}$.

THÉORÈME 5.5. L'opération $k \mapsto{ }^{h}\left(k^{h^{\prime}}\right)=\left({ }^{h} k\right)^{h^{\prime}}={ }^{h} k^{h^{\prime}}$ définit un foncteur $F \mathbf{H} \rightarrow \mathscr{G}_{r}$ tel que $h \mapsto \mathbf{K}_{p h}$. 
THÉORÈME 5.6. On a $k^{\prime}\left[h^{\prime}\right] k[h]=k^{\prime \prime}\left[h^{\prime} h\right]$ avec:

$$
k^{\prime \prime}=\left({ }^{\prime}\left[h^{\prime}\right] k\right)\left(k^{\prime h}\right)=\left(k^{\prime k[h]}\right)\left(h^{\prime} k\right),
$$

quand ces expressions ont un sens.

Proposition 5.7. Si on a une opération de $\mathbf{K} \operatorname{sur} \mathbf{H}$ :

$$
\mathbf{K}_{f} \times p^{-1}(f) \longrightarrow p^{-1}(f):(k, h) \mapsto k[h]
$$

telle que:

a) (i) de 5.1,

b) on a un foncteur $F \mathbf{H} \stackrel{\Phi}{\longrightarrow} \mathscr{G} r$ tel que

$$
h \mapsto \mathbf{K}_{p h} \quad \text { et } \quad(u, v) \mapsto{ }^{u}()^{v},
$$

c) 5.6 ,

d) ( $\star$ de 5.2 .

Alors l'opération est fidèle.

Remarque. Dans la définition 5.2 d'une panextension on peut donc remplacer (ii) par la donnée d'un foncteur $F \mathbf{H} \rightarrow \mathscr{G}_{r}$ vérifiant la propriété du théorème 5.6. Ceci justifie la seconde assertion des exemples ci-dessus.

Définition 5.8. Soit $D: F \mathbf{C} \rightarrow \mathscr{G} r$ un foncteur, on appelle panextension de $\mathbf{C}$ par $D$ une panextension $\mathbf{K} \rightarrow \mathbf{H} \stackrel{p}{\longrightarrow} \mathbf{C}$ telle que

$$
\mathbf{K}_{f}=D(f) \quad \text { et } \quad{ }^{h} k^{h^{\prime}}=D\left(p h, p h^{\prime}\right)(k) .
$$

On a ici encore la notion de section s d'une panextension comme en 2.1. En associant à chaque $f \in \mathbf{C}_{1}=(F \mathbf{C})_{0}$ le groupe $\mathbf{K}_{f}$ et à chaque $(u, v) \in(F \mathbf{C})_{1}$ le morphisme ${ }^{s(u)}()^{s(v)}$, on définit un coefficient, au sens de [9], de $F \mathbf{C}$ vers $\mathscr{G}_{r}$. Ce n'est pas nécessairement un foncteur car $s$ n'en est pas nécessairement un.

THÉORÈme 5.9. La donnée d'une section s d'une panextension $\mathbf{E}$ définit une application $\psi: \mathbf{C}_{2} \rightarrow \mathbf{K}_{1}$ telle que:

a) $\psi\left(c, c^{\prime}\right) \in \mathbf{K}_{c c^{\prime}}$ et est une identité si c ou c' en est une.

b) étant donné $\left(c, c^{\prime}, c^{\prime \prime}\right) \in \mathbf{C}_{3}$, on a la relation:

$$
{ }^{s(c)} \psi\left(c^{\prime}, c^{\prime \prime}\right) \psi\left(c, c^{\prime} c^{\prime \prime}\right)=\left(\psi\left(c, c^{\prime}\right)^{s\left(c^{\prime \prime}\right)}\right) \psi\left(c c^{\prime}, c^{\prime \prime}\right),
$$


c) étant donnés $\left(c, c^{\prime}\right) \in \mathbf{C}_{2}, k \in \mathbf{K}_{1}$ et $h \in \mathbf{H}_{1}$, on a les relations:

$$
\begin{aligned}
& s(c) s\left(c^{\prime}\right) k=\left(\psi\left(c, c^{\prime}\right)^{k[h]}\right)\left(^ { s ( c c ^ { \prime } ) } k \left(\left(\psi\left(c, c^{\prime}\right)^{-1}\right)^{h},\right.\right. \\
& k^{s(c) s\left(c^{\prime}\right)}=\left({ }^{k[h]} \psi\left(c, c^{\prime}\right)\right)\left(k^{s\left(c c^{\prime}\right)}\right)\left({ }^{h}\left(\psi\left(c, c^{\prime}\right)^{-1}\right)\right),
\end{aligned}
$$

quand ces expressions ont un sens.

DÉfINITION 5.10. L'application $\psi$ est appelée le 2-cocycle associé à la panextension $\mathbf{E}$ par la section $s$.

THÉORÈme 5.11. Soient $\psi$ et $\psi^{\prime}$ les 2-cocycles associés à $\mathbf{E}$ par des sections $s$ et $s^{\prime}$. Il existe une application $\tau: \mathbf{C}_{1} \rightarrow \mathbf{K}_{1}$ telle que

a) $\tau(c) \in \mathbf{K}_{c}$,

b) $\psi\left(c, c^{\prime}\right)=\tau\left(c c^{\prime}\right)^{-1} \psi^{\prime}\left(c, c^{\prime}\right)\left(\tau(c)^{s^{\prime}\left(c^{\prime}\right)}\right)\left(^{s(c)} \tau\left(c^{\prime}\right)\right)$

$$
=\tau\left(c c^{\prime}\right)^{-1} \psi^{\prime}\left(c, c^{\prime}\right)\left({ }^{s^{\prime}(c)} \tau\left(c^{\prime}\right)\right)\left(\tau(c)^{s\left(c^{\prime}\right)}\right) \quad \forall\left(c, c^{\prime}\right) \in \mathbf{C}_{2},
$$

c) ${ }^{s^{\prime}(c)} k=\left(\tau(c)^{k[h]}\right)\left({ }^{s(c)} k\right)\left(\tau(c)^{-1}\right)^{h}$ et $k^{s^{\prime}(c)}=\left({ }^{k[h]} \tau(c)\right)\left(k^{s(c)}\right)\left({ }^{h}\left(\tau(c)^{-1}\right)\right)$,

quand ces expressions ont un sens.

Les conditions c) de 5.9 et 5.11 se ramènent à des conditions de commutativité quand $\mathbf{E}$ est une panextension de $\mathbf{C}$ par un foncteur $D: F \mathbf{C} \rightarrow \mathscr{G} r$ et disparaît si $D$ est à valeurs dans $\mathscr{A} b$ i.e. si $D$ est un $\mathbf{C}$-système linéaire au sens de [2].

Définition 5.12. Deux panextensions de catégories $\mathbf{E}: \mathbf{K} \rightarrow \mathbf{H} \stackrel{p}{\longrightarrow} \mathbf{C}$ et $\mathbf{E}^{\prime}: \mathbf{K} \rightarrow \mathbf{H}^{\prime} \stackrel{p^{\prime}}{\longrightarrow} \mathbf{C}^{\prime}$ sont dites congrues s'il existe un foncteur inversible $\mu: \mathbf{H} \rightarrow \mathbf{H}^{\prime}$ tel que

a) $p^{\prime} \mu=p$,

b) $k[\mu(h)]=\mu(k[h])$ quand ceci a un sens.

On notera Panext $(\mathbf{C}, \mathbf{K})$, resp. Panext $(\mathbf{C}, D)$, l'ensemble des classes de congruence de panextensions de $\mathbf{C}$ par un groupoïde $\mathbf{K}$, resp. un foncteur $D: F \mathbf{C}$ $\rightarrow \mathscr{G}_{r}$.

Lemme 5.13. Dans les conditions de la définition précédente, on a:

$$
{ }^{h} k^{h^{\prime}}={ }^{\mu(h)} k^{\mu\left(h^{\prime}\right)}
$$

quand ceci a un sens. Cette propriété est triviale dans le cas où $\mathbf{E}$ est une panextension par un foncteur $D: F \mathbf{C} \rightarrow \mathscr{G}_{r}$.

ThÉORÈme 5.14. Soient $\mathbf{E}$ et $\mathbf{E}^{\prime}$ deux panextensions de $\mathbf{C}$ par $\mathbf{K}$. Si $\mathbf{E}$ et $\mathbf{E}^{\prime}$ sont congrues et si $\psi$ est un 2-cocycle associé à $\mathbf{E}$, alors $\psi$ est aussi un 
2-cocycle associé à $\mathbf{E}^{\prime}$. Réciproquement, si un même 2-cocycle $\psi$ est associé $\grave{a} \mathbf{E}$ et à $\mathbf{E}^{\prime}$, alors $\mathbf{E}$ et $\mathbf{E}^{\prime}$ sont congrues.

\section{Cohomologie à coefficients panvariants}

Considérons une (petite) catégorie $\mathbf{C}$, un foncteur $D: F \mathbf{C} \rightarrow \mathscr{G} r$ et le groupoïde

$$
\mathbf{K}=\coprod_{f \in \mathbf{C}_{1}} D(f) .
$$

On notera $\mathbf{K}_{f}=D(f)$ et ${ }^{c} k^{c^{\prime}}=D\left(c, c^{\prime}\right)(k)$.

Proposition 6.1. Si l'on a une panextension de $\mathbf{C}$ par D, alors $D$ est un C-système naturel.

Dans la suite on considère un $\mathbf{C}$-système naturel $D$.

Définition 6.2. Un 2-cocycle de $C$ vers $D$ est la donnée d'une application $\psi: \mathbf{C}_{2} \rightarrow \mathbf{K}_{1}$ telle que:

a) $\psi\left(c, c^{\prime}\right) \in \mathbf{K}_{c c^{\prime}}$ et est une identité si $c$ ou $c^{\prime}$ en est une,

b) ${ }^{c} \psi\left(c^{\prime}, c^{\prime \prime}\right) \psi\left(c, c^{\prime} c^{\prime \prime}\right)=\left(\psi\left(c, c^{\prime}\right)^{c^{\prime \prime}}\right) \psi\left(c c^{\prime}, c^{\prime \prime}\right) \quad \forall\left(c, c^{\prime}, c^{\prime \prime}\right) \in \mathbf{C}_{3}$.

DÉFInition 6.3. Deux cocycles $\psi$ et $\psi^{\prime}$ de $\mathbf{C}$ vers $D$ sont dits cohomologues s'il existe une application $\tau: \mathbf{C}_{1} \rightarrow \mathbf{K}_{1}$ telle que:

a) $\tau(c) \in \mathbf{K}_{c}$,

b) $\psi\left(c, c^{\prime}\right)=\tau\left(c c^{\prime}\right)^{-1} \psi^{\prime}\left(c, c^{\prime}\right)\left(\tau(c)^{c^{\prime}}\right)\left({ }^{c} \tau\left(c^{\prime}\right)\right)$

$$
=\tau\left(c c^{\prime}\right)^{-1} \psi^{\prime}\left(c, c^{\prime}\right)\left({ }^{c} \tau\left(c^{\prime}\right)\right)\left(\tau(c)^{c^{\prime}}\right), \quad \forall\left(c, c^{\prime}\right) \in \mathbf{C}_{2} .
$$

DÉfinition 6.4. L'ensemble des classes de cohomologie de 2-cocycles de $\mathbf{C}$ vers $D$ est appelé l'ensemble de cohomologie de dimension 2 de $\mathbf{C}$ vers $D$ et est noté $H^{2}(\mathbf{C}, D)$.

THÉorème 6.5. L'application, qui à chaque panextension de $\mathbf{C}$ par $D$ associe la classe de cohomologie d'un de ses 2-cocycles associés, définit une bijection

$$
\omega: \operatorname{Panext}(\mathbf{C}, D) \longrightarrow H^{2}(\mathbf{C}, D) \text {. }
$$

Compte tenu de 5.7, les panextensions de $\mathbf{C}$ par $D$ sont exactement les extensions linéaires de $\mathbf{C}$ par $D$ et le dernier théorème se trouve dans [2]. On comparera sa démonstration et celle de 4.6.

La théorie de Baues-Wirsching que l'on retrouve ici est reliée par Pavešić dans [12] à l'extension de la cohomologie de Hochschild décrite par Gerstenhaber-Schack dans [5]. 


\section{RÉFÉRENCES}

1. Baues, H. J., and Minian, E. G., Track extensions of categories and cohomology, K-theory 23 (2001), 1-13.

2. Baues, H. J., and Wirsching, G., Cohomology of small categories, J. Pure Appl. Algebra 38 (1985), 187-211.

3. Datuashvili, T., On the cohomology of categories (russian), Trudy Tbiliss. Mat. Inst. 62 (1979), $28-37$.

4. Dedecker, P., and Lue, A. T. S., A non abelian two-dimensional cohomology for associative algebras, Bull. Amer. Math. Soc. 72 (1966), 1044-1050.

5. Gerstenhaber, M., and Schack, S., On the deformation of algebra morphisms and diagrams, Trans. Amer. Math. Soc. 279 (1983), 1-50.

6. Golasinski, M., $n$-fold extensions and cohomology of small categories, Mathematica (Cluj) 31 (54) (1989), 53-59.

7. Hochschild, G., On the cohomology groups of an associative algebra, Ann. of Math. 46 (1945), 58-67.

8. Hoff, G., On the cohomology of categories, Rend. Mat. 7 (1974), 169-192.

9. Hoff, G., Cohomologies et extensions de catégories, Math. Scand. 74 (1994), 191-207.

10. Lue, A. T. S., Non-abelian cohomology of associative algebras, Quart. J. Math. Oxford 19 (1968), 159-180.

11. Mitchell, B., Rings with several objects, Adv. Math. 8 (1972), 1-161.

12. Pavešić, P., Diagram cohomologies using categorical fibrations, J. Pure Appl. Algebra 112 (1996), 73-90.

LAGA (UMR 7539 DU CNRS)

INSTITUT GALILÉE

AVENUE J. B. CLÉMENT

F-93430 VILLETANEUSE

FRANCE

E-mail: hoff@math.univ-paris13.fr 